\title{
Alterações no volume nasal de pacientes submetidos a disjunção da maxila
}

\author{
Renata da Fonseca Lacerda e Muniz*, Mario Cappellette Jr.**, Daniela Carlini***
}

\begin{abstract}
Resumo
Os efeitos da disjunção maxilar na resistência nasal e fluxo aéreo têm sido amplamente discutidos na literatura, com controvérsias. Suas indicações esqueléticas e dentárias parecem estar bem claras. Porém, aquelas puramente rinológicas não são justificadas, porque nem sempre resultados positivos são encontrados. Este estudo teve por finalidade avaliar a repercussão da disjunção maxilar ortopédica no aspecto respiratório e rinológico dos pacientes submetidos a esse procedimento.
\end{abstract}

Palavras-chave: Expansão rápida da maxila. Resistência nasal. Atresia maxilar.

\section{INTRODUÇÃO}

A revisão da literatura mostra que a disjunção maxilar tem sido empregada há mais de 100 anos, objetivando o aumento em largura do arco maxilar que se apresenta constrito.

Esse método de tratamento foi descrito pela primeira vez na literatura em 1860, por E. H. Angell ${ }^{1}$, que o empregou com a finalidade de solucionar problemas de apinhamento no arco dentário superior de uma menina de 14 anos de idade $^{1}$.

Seus efeitos, por meio da abertura da sutura palatina mediana, são descritos como não somente ortodônticos e ortopédicos, mas podendo influenciar também a cavidade nasal, por causa da sua íntima relação com a estrutura maxilar ${ }^{2,5,6,7,8,}$ $9,10,11,12,13,17,18,19,20,21$.
A partir dessa constatação, inúmeras pesquisas têm sido desenvolvidas com o propósito de avaliar os possíveis efeitos da disjunção maxilar sobre a resistência e fluxo aéreo nasal.

A literatura mostra que os resultados são controversos. Alguns autores ${ }^{3,4,6,11}$ defendem com entusiasmo o seu emprego, afirmando que ocorrem, além de uma melhora na configuração do arco maxilar, também uma redução na resistência nasal, aumento no fluxo aéreo e até mesmo uma mudança favorável no modo de respiração, enquanto outros autores ${ }^{7,9,10}$ continuam céticos em relação a essas alterações.

Neste estudo, foi feita uma revisão bibliográfica, com o objetivo de buscar dados confiáveis e bem documentados, a respeito das alterações na permeabilidade nasal em pacientes submetidos à

* Cirurgiã-Dentista, Especialista em Ortodontia e Ortopedia Facial pela ABO - SP.

** Cirurgião-Dentista, Especialista em Radiologia pela USF, Mestre em Ortodontia e Ortopedia Facial pela UNICASTELO, Doutor em Ciências pela Escola Paulista de Medicina - UNIFESP e Coordenador do Curso de Especialização em Ortodontia e Ortopedia Facial da ABO - SP e FAMOSP.

*** Médica, Mestre e Doutora em Otorrinolaringologia pela Escola Paulista de Medicina - UNIFESP. 
disjunção maxilar.

Entre os dados obtidos na literatura pesquisada, destacam-se: alterações na dimensão da cavidade nasal, alterações na resistência nasal ao fluxo aéreo, alterações no fluxo aéreo nasal, exames empregados para as avaliações, indicação da disjunção maxilar somente para fins respiratórios.

\section{Alterações na dimensão da cavidade nasal}

Pullen ${ }^{15}$ afirmou que por causa interdependência entre arco maxilar e estrutura nasal, o procedimento da disjunção maxilar pode oferecer um estímulo de desenvolvimento às estruturas nasais localizadas logo acima da maxila.

Com o uso de um crânio, Brown ${ }^{2}$, demonstrou que a força aplicada no palato pode separar a sutura palatina mediana em toda a sua extensão, e também a sutura intermaxilar, e que então a largura das narinas poderia ser aumentada. Observou em seus pacientes que após a disjunção maxilar, houve aumento de espaço para a respiração nasal, com conseqüente melhora na saúde dos tecidos intranasais e em sua saúde geral.

Avaliando telerradiografias em norma frontal de 10 pacientes submetidos à disjunção maxilar, Haas $^{5}$ observou aumentos nas cavidades nasais que variaram de $2 \mathrm{~mm}$ a $4,5 \mathrm{~mm}$ em 12 a 27 dias de tratamento.

Segundo afirmação de $\mathrm{Wertz}^{19}$, a cavidade nasal pode ser aumentada após a disjunção maxilar somente em sua porção ântero-inferior, em conseqüência da configuração anatômica das estruturas das paredes laterais do nariz e da alteração espacial destas, resultante da disjunção maxilar, uma vez que a maior abertura da sutura palatina mediana ocorre na região anterior do palato.

Hershey et al. ${ }^{8}$, encontraram aumentos na área nasal transversal, que variaram de $1 \mathrm{~mm}$ a $3 \mathrm{~mm}$ em sua amostra de 17 pacientes submetidos à disjunção maxilar. $\mathrm{O}$ valor médio da área nasal transversal logo após a disjunção foi encontrado em $28,88 \mathrm{~mm}$, considerado significante quando comparado com o valor médio inicial, de 26,76mm.

Warren et al. ${ }^{18}$, compararam a área nasal transversal de pacientes tratados com disjunção maxilar ortopédica e disjunção maxilar cirurgicamente assistida com grupos controle juvenil e adulto, respectivamente. Constataram um aumento na dimensão da cavidade nasal de aproximadamente $45 \%$ após a disjunção maxilar ortopédica, e de $55 \%$ após a disjunção maxilar cirúrgica.

Analisando a área nasal transversal de uma paciente de 8,9 anos de idade submetida à disjunção maxilar, Marchioro et al. ${ }^{11}$, em 1997, constataram aumento nessa área que passou de $0,55 \mathrm{~cm}^{2}$ (prédisjunção) para $0,61 \mathrm{~cm}^{2}$ (pós- disjunção).

Diferentemente de Wertz ${ }^{19}$ e Paiva ${ }^{14}$, em seu estudo com 25 pacientes submetidos a tratamento com disjunção maxilar, observou por meio do exame da nasofibroscopia um afastamento lateral da extremidade posterior da concha nasal inferior que levou a um aumento transversal da região posterior da cavidade nasal, com conseqüente aumento de $10 \%$ do espaço livre da nasofaringe.

Hahn et al. ${ }^{6}$, em sua amostra de 27 pacientes submetidos à disjunção maxilar, previamente ao exame de rinometria acústica, fizeram o uso de descongestionante nasal para eliminar quaisquer interferências que pudessem influenciar os resultados do exame. Observaram que 100\% dos pacientes mostraram aumento do volume da cavidade nasal entre os períodos pré-disjunção e logo após a disjunção maxilar.

Marchioro et al. ${ }^{12}$, encontraram aumento na área nasal transversal, após o uso de descongestionante, de 17,5\% em 24 indivíduos (88,89\%) de sua amostra de 27 pacientes submetidos à disjunção maxilar, valor que se manteve estável 90 dias após a disjunção maxilar. Os aumentos observados nas dimensões da cavidade nasal mostraramse ainda maiores após o período de contenção na amostra de Hershey et al. ${ }^{8}$ (1976), cujo aumento médio passou de $26,76 \mathrm{~mm}$ na avaliação imediatamente pós-disjunção para 29,23mm após o 
período de contenção, sendo justificado pela força residual do aparelho disjuntor e nos ossos do terço médio da face. Na paciente observada por Marchioro et al. ${ }^{11}$, a área nasal transversal passou de $0,61 \mathrm{~cm}^{2}$ imediatamente após a disjunção para $0,7 \mathrm{~cm}^{2} 90$ dias após o final da disjunção maxilar. Ao contrário, após o período de contenção, Hahn et al. ${ }^{6}$ (1999) e Marchioro et al. ${ }^{12}$ observaram discreta diminuição do volume da cavidade nasal, não significativa estatisticamente.

Em estudo sobre o volume nasal em 50 pacientes respiradores orais com idade entre 4 e 14 anos, Cappellette $\mathrm{Jr}^{3}{ }^{3}$, em 2005, concluiu que a disjunção maxilar promoveu um aumento do volume nasal e da área transversal mínima estatisticamente significantes.

Em 2006, Cappellette Jr. et al. ${ }^{4}$, em estudo com rinometria acústica em crianças de 7 e 8 anos de idade submetidas à disjunção maxilar, constataram que os valores das áreas transversais mínimas e volumes nasais pré e pós disjunção maxilar, apresentaram aumento significantes concluindo assim que a rinometria acústica é um método objetivo para avaliar a variação da cavidade nasal.

\section{Alterações na resistência nasal ao fluxo aéreo}

Linder-Aronson e Aschan ${ }^{10}$, em seu estudo com 10 pacientes respiradores orais, observaram redução na resistência nasal à respiração, após tratamento com disjunção maxilar. A resistência nasal foi medida usando a técnica de Aschan, Drettner e Ronge, que consiste do registro simultâneo da pressão de ar entre a nasofaringe e as narinas, assim como o valor do fluxo aéreo.

Essa mensuração foi feita antes, imediatamente após e 1 ano após a disjunção maxilar, com e sem o uso de descongestionante nasal. Os valores da resistência nasal obtidos antes do uso de descongestionante foram considerados como resistência funcional, e aqueles obtidos após o uso de descongestionante foram considerados como resistência anatômica. Uma redução significante da resistência nasal foi observada após a disjunção maxilar, com ou sem o uso de descongestionante, aproximando-se de valores compatíveis com os de respiração nasal, segundo os autores. Os resultados se mostraram estáveis 1 ano após o tratamento.

Wertz $^{19}$, observou aumento da permeabilidade nasal em um grupo de 9 pacientes submetidos à disjunção maxilar, quando avaliados durante máximo esforço respiratório. A melhora variou de leve a muito significante e mostrou-se estável 3 meses após o tratamento.

Hershey et al. ${ }^{8}$, obtiveram redução de $45 \%$ na resistência nasal de sua amostra de 17 pacientes respiradores orais submetidos à disjunção maxilar, se aproximando a níveis compatíveis com aqueles de respiração nasal e significantemente mais baixos que a resistência nasal de respiradores orais, segundo os autores. A melhora se manteve estável 3 meses após a disjunção maxilar. Para a avaliação foram usados dois cateteres, um deles inserido na orofaringe o mais posteriormente possível e outro adaptado em uma máscara nasal. Com o recurso, os autores obtiveram valores de pressão e fluxo aéreo para então determinar a resistência nasal por meio da equação modificada da lei de Ohm.

Hartgerink et al. ${ }^{7}$, avaliaram a resistência nasal de 38 pacientes submetidos à disjunção maxilar em 4 condições: respiração natural ou em repouso, respiração com dilatação das narinas, respiração após a administração de descongestionante nasal, respiração após o uso de descongestionante com dilatação nasal. Obtiveram redução da resistência nasal em 65\% dos pacientes avaliados em estado natural, 56\% com dilatação das narinas, 39\% com uso de descongestionante e 35\% com descongestionante e dilatação das narinas. As alterações mostraram-se estáveis 1 ano após a disjunção maxilar. Os autores consideraram grande o número de pacientes que não mostraram redução na resistência nasal, atribuindo o fato 
a uma alta variabilidade individual à resposta à disjunção maxilar.

Marchioro et al. ${ }^{11}$, observaram redução significante da resistência nasal de uma paciente de 8,9 anos de idade submetida à disjunção maxilar. Os valores da resistência nasal foram avaliados por meio da rinomanometria ativa anterior antes da disjunção maxilar, imediatamente após e 90 dias após o tratamento, com administração de descongestionante nasal, e mostraram-se estáveis 3 meses após a disjunção.

Rizzatto et al. ${ }^{16}$, avaliaram a resistência nasal de uma amostra de 26 pacientes submetidos à disjunção maxilar. Os valores da resistência nasal foram avaliados por meio da rinomanometria ativa anterior, antes da disjunção maxilar, imediatamente após e 90 dias após o tratamento com administração de descongestionante nasal. Como resultado obtiveram redução significativa de $37,87 \%$ na resistência nasal média imediatamente após a disjunção maxilar, que se manteve estável após 90 dias de contenção. A diminuição na resistência nasal foi observada em 22 pacientes imediatamente após a disjunção maxilar e em 21 pacientes 90 dias após o tratamento.

\section{Alterações no fluxo aéreo nasal}

O fluxo aéreo nasal aumentou após a disjunção maxilar, segundo as observações de Brown ${ }^{2}$, que em sua prática clínica obteve relatos de seus pacientes reconhecendo diferenças na respiração nasal, sendo a melhora uma indicação de que houve ampliação da cavidade nasal e aumento do volume de ar a cada inalação. O grau dessa alteração, segundo o autor, é governado naturalmente pela condição da membrana da mucosa nasal, a qual pode ser rápida, lenta ou variável em sua resposta.

Wertz $^{19}$, mediu o fluxo aéreo nasal antes, imediatamente após e 3 meses após a disjunção maxilar em dois grupos de pacientes. No grupo I, composto de 4 pacientes, o fluxo aéreo foi medido quando em repouso e apenas 1 paciente mostrou aumento no fluxo aéreo, enquanto os outros 3 mostraram diminuição. No grupo II, 9 pacientes foram avaliados quando em repouso, após exercício leve e após máximo esforço respiratório. Antes das mensurações foi administrado descongestionante nasal com o intuito de reduzir ao mínimo as interferências dos tecidos intranasais. Quando avaliados em repouso, 7 pacientes mostraram aumento no fluxo aéreo, enquanto 2 mostraram diminuição e após exercícios leves, 6 pacientes mostraram aumento e 3 mostraram diminuição do fluxo aéreo.

Normando et al. ${ }^{13}$, obtiveram aumento de $50 \mathrm{ml}$ no fluxo aéreo nasal de uma paciente submetida à disjunção maxilar quando avaliada em estado natural ou em repouso. Quando foi solicitado à paciente uma inspiração ou expiração forçada, não foi constatado aumento no fluxo aéreo.

Resultados diferentes foram obtidos por Pai$\mathrm{va}^{14}$, que avaliando 25 pacientes tratados com disjunção maxilar, avaliados quanto ao fluxo aéreo nasal por meio da rinomanometria em estado natural, após o uso de solução vasoconstritora para evitar interferências dos tecidos intranasais, não obteve alteração significante do fluxo aéreo após a disjunção maxilar quando comparado com aquele da fase pré-disjunção.

\section{Exames empregados nas avaliações}

Para avaliação das dimensões da cavidade nasal, a maioria dos autores escolheu o exame de rinometria acústica ${ }^{3,4,6,11,12,20}$. Para o mesmo fim, Paiva ${ }^{14}$ escolheu a nasofibroscopia.

A avaliação do fluxo aéreo nasal foi feita por meio da rinomanometria ativa anterior ${ }^{11,14,16}$, ou por meio da espirometria nasal ${ }^{13}$.

\section{Indicação da disjunção maxilar somente para fins respiratórios}

O emprego da disjunção maxilar somente para fins respiratórios não se justifica ${ }^{7,16}$, a menos que a obstrução que causa a estenose nasal esteja 


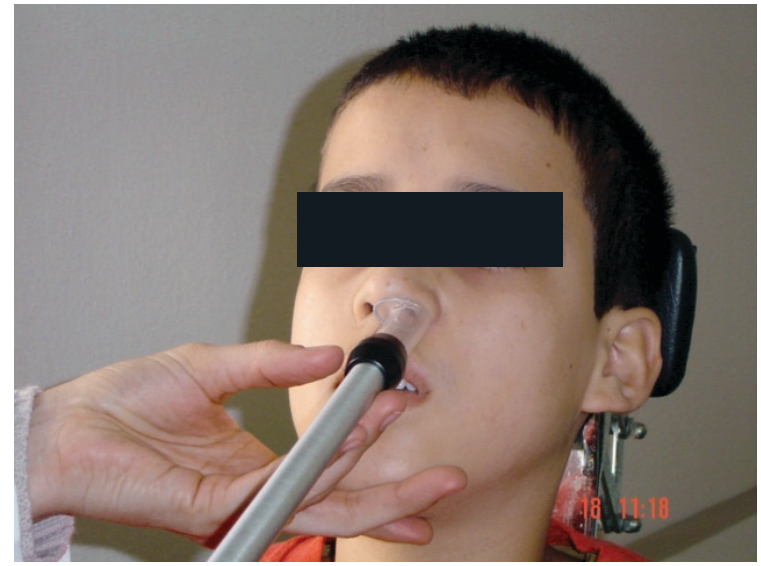

FIGURA 1 - Exame de rinometria acústica.

localizada na porção ântero-inferior da cavidade nasal e seja acompanhada por constrição maxi$\operatorname{lar}^{19}$.

\section{CONCLUSÃO}

Após revisão da literatura a respeito das alterações na permeabilidade nasal promovidas pela disjunção maxilar, pôde-se observar que esse método de tratamento, segundo grande parte dos autores, além de melhorar a configuração do arco maxilar que se apresenta constrito, provoca também um aumento na cavidade nasal, possibilitando assim uma diminuição na resistência nasal e até mesmo aumento do fluxo aéreo.

Porém, alguns autores ainda permanecem relutantes quanto às suas indicações rinológicas, porque em suas amostras alguns pacientes não experimentaram melhoras no quadro respiratório, sendo o fato justificado pela variabilidade individual.

Conclui-se então que a disjunção maxilar tem indicações ortodônticas e ortopédicas precisas, mas as indicações para fins respiratórios ainda requerem estudos mais específicos, com amostras bem selecionadas, ressaltando que o diagnóstico do caso a ser tratado, em que se espera uma melhora da função nasal, deve sempre envolver o ortodontista e otorrinolaringologista.

Changes in nasal volume of patients undergoing rapid maxillary expansion

\begin{abstract}
Rapid maxillary expansion effects on airflow and nasal resistance has been amply discussed in literature, with controversies. Its skeletal and dental indications seem to be clear, however, those sole rhinologic are not justified, because positive results are not always found. This study had as purpose to evaluate the orthopedic rapid maxillary expansion repercussion on respiratory and rhinologic aspects of patients undergoing this procedure.
\end{abstract}

Key words: Rapid maxillary expansion. Nasal resistance. Maxillary constriction. 


\section{REFERÊNCIAS}

1. ANGELL, E. H. Treatment of irregularity of the permanent or adult teeth. Part I. Dental Cosmos, Philadelphia, v. 1, no. 10, p. 540-544, May 1860.

2. BROWN, V. I. The pathologic and therapeutic possibilities of upper maxillary contraction and expansion. Dental Cosmos, Philadelphia, v. 56, no. 2, p. 137-154, Feb. 1914.

3. CAPPELLETTE JÚNIOR, M. Avaliação de volume nasal pré e pós disjunção maxilar ortopédica. 2005. Tese (Doutorado)Escola Paulista de Medicina da USP, São Paulo, 2005.

4. CAPPELLETTE JR., M.; CARLINI D.; PIGNATARI, S. S. N.; CRUZ, O. L. M.; WECKX, L. L. M. Rinometria acústica em crianças submetidas à disjunção maxilar. Rev. Dental Press Ortodon. Ortop. Facial, Maringá, v. 11, n. 2, p. 84-92, mar./abr. 2006.

5. HAAS, A. J. Rapid expansion of the maxillary dental arch and nasal cavity by opening the midpalatal suture. Angle Orthod. Appleton, v. 31, no. 2, p. 73-89, Apr. 1961.

6. HAHN, L.; MARCHIORO, E. M.; RIZZATTO, S. D.; ROITHMANN, R.; COSTA, N. P. Avaliação do volume da cavidade nasal antes e após a expansão rápida da maxila por meio da rinometria acústica. Ortodontia Gaúcha, Porto Alegre, v. 3, n. 2, p. 85-96, jul./dez. 1999.

7. HARTGERINK, D. V.; VIG, P. S.; ABBOTT, D. W. The effect of maxillary expansion on nasal airway resistance. Am. J. Orthod. Dentofacial Orthop., St. Louis, v. 92, no. 5, p. 381-389, 1987.

8. HERSHEY, H. G.; STEWART, B. L.; WARREN, D. W. Changes in nasal airway resistance associated with rapid maxillary expansion. Am. J. Orthod., St. Louis, v. 69, no. 3, p. 274-284, Mar. 1976.

9. KLUEMPER, G. T.; VIG, P. S.; VIG, K. W. L. Nasorespiratory characteristics and craniofacial morphology. Eur. J. Orthod., Oxford, v. 17, no. 6, p. 491-495, Dec. 1995

10. LINDER-ARONSON, S.; ASCHAN, G. Nasal Resistance to breathing and palatal height before and after expansion of the median palatine suture. Odontol. Revy, Lund, v. 14, no. 3, p. 254-270, 1963.

11. MARCHIORO, E. M.; RIZZATTO, S. D.; ROITHMANN, R.; LUBIANCA NETO, J. F. O efeito da expansão rápida da maxila na geometria e função nasal: relato de caso. Ortodontia Gaúcha, Porto Alegre, v. 1, n. 1, p. 3-7, abr. 1997.
12. MARCHIORO, E. M.; MARTINS, J. R.; ROITHMANN, R.; RIZZATTO, S.; HAHN, L. Efeito da expansão rápida da maxila na cavidade nasal avaliado por rinometria acústica. Rev. Dental Press Ortodon. Ortop. Facial, Maringá, v. 6, n. 1, p. 31-38, jan./fev. 2001.

13. NORMANDO, A. D. C.; RIBEIRO, K. C. F.; SOTÃO, A. B.; NORMANDO, V. M. F. Os efeitos da expansão rápida da maxila sobre o fluxo aéreo nasal - revisão da literatura e apresentação de um caso clínico. Rev. Paran. Odontol., Curitiba, v. 1, n. 2, p. 19-26, jul./dez. 1996.

14. PAIVA, J. B. Estudo rinomanométrico e nasofibroendoscópico da cavidade nasal dos pacientes submetidos à expansão rápida da maxila. 1999. Tese (Doutorado)-Faculdade de Odontologia da Universidade de São Paulo, São Paulo, 1999.

15. PULLEN, H. A. Expansion of the dental arch and opening the maxillary suture in relation to the development of the internal and external face. Dental Cosmos, Philadelphia, v. 54, no. 5, p. 509-524, May 1912.

16. RIZZATTO, S. M. D.; COSTA, N. P.; MARCHIORO, E. M.; SAFFER, M. Avaliação do efeito da expansão rápida da maxila na resistência nasal por rinomanometria ativa anterior em crianças. Ortodontia Gaúcha, Porto Alegre, v. 2, n. 2, p. 79-93, jul./dez. 1998.

17. TIMMS, D. J. Some medical aspects of rapid maxillary expansion. Br. J. Orthod., Oxford, v. 1, no. 4, p. 127-132, July 1974.

18. WARREN, D. W.; HERSHEY, H. G.; TURVEY, T. A.; HINTON, V. A.; HAIRFIELD, W. M. The nasal airway following maxillary expansion. Am. J. Orthod. Dentofacial Orthop., St. Louis, v. 91, no. 2, p. 111-116, Feb. 1987.

19. WERTZ, R. A. Changes in nasal airflow incident to rapid maxillary expansion. Angle Orthod., Appleton, v. 38, no. 1, p. 1-11, Jan. 1968.

20. ZAVRAS, A. I.; WHITE, G. E.; RICH, A.; JACKSON, A. C. Acoustic rhinometry in the evaluation of children with nasal or oral respiration. J. Clin. Pediatr. Dent., Birmingham, v. 13, no. 3, p. 203-210, 1994.
Endereço para correspondência

Renata da Fonseca Lacerda e Muniz

Av. Jurema, 147 apto 102

CEP: 04.079-000 - Moema - São Paulo / SP

E-mail: rfmuniz@hotmail.com 